\title{
Endobronchial ultrasound: morphological predictors of benign disease
}

\author{
Pratibha Gogia ${ }^{1}$, Tabassum Z. Insaf ${ }^{2}$, William McNulty ${ }^{1,3}$, Afroditi Boutou ${ }^{1}$, \\ Andrew G. Nicholson ${ }^{1,4}$, Zaid Zoumot ${ }^{1,5,6}$ and Pallav L. Shah ${ }^{1,3,6}$
}

Affiliations: ${ }^{1}$ The NIHR Respiratory Biomedical Research Unit at the Royal Brompton and Harefield National Health Service (NHS) Foundation Trust and Imperial College, London, UK. ${ }^{2}$ School of Public Health University at Albany, State University of New York, Rensselaer, NY, USA. ${ }^{3}$ Chelsea and Westminster Hospital NHS Foundation Trust, London, UK. ${ }^{4}$ Dept of Histopathology, Royal Brompton and Harefield NHS Foundation Trust, London, UK. ${ }^{5}$ Respiratory and Critical Care Institute, Cleveland Clinic Abu Dhabi, Abu Dhabi, United Arab Emirates. ${ }^{6}$ These authors contributed equally.

Correspondence: Pallav L. Shah, Royal Brompton Hospital, Sydney Street, London SW3 6NP, UK. E-mail: pallav.shahdimperial.ac.uk

ABSTRACT The objective of this study was to assess the utility of endobronchial ultrasound (EBUS) morphology of lymph nodes in predicting benign cytology of transbronchial needle aspirates in a prospective observational study.

Five ultrasonic morphological characteristics of mediastinal and hilar lymph nodes were recorded: size, shape, margins, echogenic appearance and the presence of a central blood vessel. These characteristics were correlated with the final diagnosis. A total of 402 consecutive patients (237 males and 165 females) undergoing EBUS were studied.

The final diagnosis was malignant disease in $244(60.6 \%)$ and benign disease in $153(38.05 \%)$ subjects. Out of 740 sampled nodes, in $463(62.6 \%)$ malignant cells were identified, whereas in 270 (36.5\%) nodes, no malignant cells were identified. On univariate analysis small size, triangular shape and the presence of a central vessel were predictive of a benign aetiology. In the final multivariate model, a predictive probability of 0.811 (95\% CI $0.72-0.91)$ for benign disease was found if lymph node size was $<10 \mathrm{~mm}$ and a central vessel was present.

Sonographic appearances of lymph nodes improve the predictive probability of EBUS for benign aetiologies, and may reduce the number of nodes requiring sampling and the need for further invasive investigations.

@ERSpublications

Node size $<10 \mathrm{~mm}$, central vessels and triangular shape on EBUS increase confidence in cytology of a benign aetiology http://ow.ly/V1Ewh

Received: Aug 052015 | Accepted after revision: Nov 162015

Support statement: P. Gogia was on a short-term European Respiratory Society fellowship at Royal Brompton Hospital, London, UK, under P.L. Shah. Funding information for this article has been deposited with FundRef.

Conflict of interest: None declared.

Copyright $\odot$ ERS 2015. This article is open access and distributed under the terms of the Creative Commons Attribution Non-Commercial Licence 4.0. 


\section{Introduction}

Endobronchial ultrasound (EBUS) has enhanced the field of vision for the bronchoscopist, from within the airways to beyond the airway wall. It scans parallel to the insertion direction of the bronchoscope with a penetration depth of $2-5 \mathrm{~cm}$ and provides a long axis image of surrounding structures in proximity to the major airways. EBUS is a minimally invasive procedure that allows sampling of lymph nodes and any masses adjacent to the airway [1] but the value of the sonographic appearances of lymph nodes remains unknown. Sonographic features are useful imaging tools in the evaluation of cervical and axillary lymph node metastasis in head and neck cancers and breast cancers, but similar features have not been sufficiently validated in EBUS $[2,3]$. A strong likelihood of benign diseases on the basis of endosonographic features may reduce the need to proceed to more invasive sampling in selected clinical scenarios.

For disease staging in lung cancer, the yield and outcomes are superior when EBUS-guided transbronchial needle aspiration (TBNA) is the initial investigation [4, 5]. Tremblay et al. [6] and Von Bartheld et al. [7] have also shown that EBUS is more efficacious at detecting granulomas than conventional bronchoscopy in patients with stage I/II sarcoidosis.

We prospectively analysed the endosonographic features of consecutive patients undergoing EBUS and correlated the findings with the final diagnosis to determine the likelihood of benign diseases.

\section{Materials and methods}

Study population

All patients who underwent EBUS between September 2007 and May 2013 at the Royal Brompton Hospital, London, UK, were evaluated. EBUS was performed for the diagnosis of mediastinal, hilar or peribronchial lymphadenopathy or masses. This included lung cancer patients undergoing staging investigations, any cancer patient with mediastinal lymphadenopathy and/or increased uptake on positron emission tomography (PET)-computed tomography (CT) (standardised uptake value $>2.5$ ), and patients with mediastinal lymphadenopathy of unknown cause. Radiologically, lymphadenopathy was defined as lymph node diameter more than $10 \mathrm{~mm}$ in the short axis $[8,9]$. In the present study, all PET-positive lymph nodes and lymph nodes documented on EBUS assessment (as small as $4 \mathrm{~mm}$ ) were assessed $[10,11]$. If EBUS cytology was inconclusive for any malignant or specific benign disease (that is, negative for malignant cells and granuloma, and mycobacterial cultures sterile), patients were counselled regarding mediastinoscopy to further try and obtain a diagnosis. If a patient did not consent to mediastinoscopy, they were followed up for at least 12 months for clinical or radiological deterioration.

Lymph node stations and numbers were determined according to the seventh edition of the International Association for the Study of Lung Cancer classification [12]. Ultrasonographic morphology of various groups of lymph nodes was categorised according to: 1) size in the short axis; 2) shape (rounded or triangular; lymph nodes were recognised as triangular if the operator could see three arms on a sonographic image); 3) margins (defined or ill-defined); 4) central blood vessel (present or absent); and 5) echographic pattern (homogeneous or heterogeneous). These morphological features were correlated with the final outcome, i.e. malignant versus nonmalignant (e.g. sarcoidosis, tuberculosis, reactive nodes or any other benign condition).

EBUS-TBNA was performed on an outpatient basis under moderate sedation (midazolam with alfentanil) with local anaesthesia. Convex probe EBUS (BF-UC260F-OL8; Olympus, Tokyo, Japan) was used for the procedure. The ultrasound features were processed by a dedicated ultrasound scanner (EU-C60; Olympus). The lymph nodes were systematically visualised. EBUS-TBNA was then performed starting with any potential N3 lymph nodes followed by N2 lymph nodes, and N1 lymph nodes. Morphological characteristics were recorded prior to needle aspiration. All morphological features were studied by two operators (P.L. Shah and Z. Zoumot).

A 22-gauge needle was used to perform TBNA (NA-201SX-4022; Olympus) for lymph node sampling. The needle, with a protective sheath, was introduced through the bronchoscope channel. The bronchoscope was manoeuvred and apposed to the airway wall at the desired location. The needle was then inserted through the airway wall into the lymph node under ultrasound guidance. The needle was advanced through different planes of the lymph node whilst constant suction pressure is applied through a $20-\mathrm{cm}^{3}$ syringe. Three passes were made at all sampled lymph node locations. Rapid onsite cytological evaluation was not available at our centre.

The aspirated material was flushed into saline, and sent for cytology and mycobacterial culture where indicated. From 2007 to 2012, Papanicolaou and Giemsa staining and light microscopy were performed by an independent pathologist who was not aware of the EBUS image characteristics. When required, cell blocks were created, fixed with formalin, and stained with haematoxylin and eosin, with immunohistochemistry and molecular analysis performed if needed. From 2012, in an effort to preserve 
tissue for those diagnosed with malignancy, samples were processed straight to cell pellets, validation studies having shown that this did not reduce the accuracy of diagnosis.

Lymph nodes were considered malignant if malignant cells were identified from EBUS aspirates or, where necessary, following surgical sampling or other radiologically guided biopsies. Where all sampling was inconclusive, the lymph nodes were considered malignant if there was fluorodeoxyglucose activity on PET scanning and evidence of disease progression over a 12-month follow-up period.

Lymph nodes where granulomatous inflammation was identified and/or where Mycobacterium tuberculosis was cultured following sampling were classified as benign disease. Lymph nodes from which adequate lymphocytes were aspirated but with no defining features were classified as benign only after further negative sampling or in the absence of clinical progression over a 12-month period. The clinical picture and PET-CT scans were also required to be consistent with benign disease.

\section{Statistical analysis}

To study the distribution of our variables of interest, we calculated univariate proportions. We calculated crude odds ratios with $95 \%$ confidence intervals and receiver operating characteristic (ROC) statistics to study the relationship between the predictor variables and benign node status [13]. We used logarithmic binomial regression to directly calculate prevalence ratios and $95 \%$ confidence intervals [13]. This method is preferable in studies of common outcomes where conventional logistic regression would overestimate relative risks. The initial multivariate model consisted of all variables that were significant at $\alpha=0.20$ in univariate analysis. We used purposeful selection with backward elimination techniques to derive the preliminary final model. A covariate was considered to be a confounder if the partial regression coefficient of other terms varied by more than $10 \%$ when the covariate was deleted from the model. Any covariate that was a confounder by this criterion was also retained and adjusted for in the model. The preliminary final model was the most prudent model, containing all significant predictors at the $\alpha=0.05$ level. We then assessed interactions between the selected predictors by introducing multiplicative terms between all final predictors. We did not find any statistically significant interactions on the multiplicative scale using this method. The model fit was assessed using deviance and Pearson statistics tests [14] as well as by plotting the ROC curve. All reported p-values are from two-sided tests of significance. We then calculated predicted probabilities for each linear combination of predictor variables from this final model. We also conducted a separate analysis using size as a continuous variable. We used the same model-building technique as detailed above for this analysis. The predicted probabilities from this model were plotted to further delineate the relationship between the size of the node and benign status.

\section{Results}

Data were collected for 402 patients: 237 males and 165 females with ages ranging from 20 to 92 years. A total of 740 lymph nodes were sampled (table 1). Three nodal stations were sampled in 79 (19\%) patients, two nodal stations were sampled in $180(46 \%)$ patients and one nodal station was sampled in 143 (35\%) patients.

No major complications associated with EBUS bronchoscopy were documented. The smallest lymph node sampled was $3 \mathrm{~mm}$ and largest was $45 \mathrm{~mm}$. The morphological characteristics of the sampled lymph nodes are detailed in table 2 . The mean \pm SD size of sampled nodes was $11.2 \pm 14 \mathrm{~mm}$ but the average size of nodes with a benign outcome was $10.5 \pm 6.4 \mathrm{~mm}$ and with a malignant outcome was $13.9 \pm 8 \mathrm{~mm}$. Size greater than

\section{TABLE 1 Demographic profile of patients and malignancy status for lymph nodes}

Total
Patients (n=402)

Lymph nodes $(n=740)$

Males $n$

Females $n$

Age years mean (range)

Final diagnosis

Malignant

Benign

Sarcoidosis

Tuberculosis

Other infection

Benign/reactive
237

165

65.7 (20-92)

244 (60.6)

153 (38.1)

31

19

3

100

Data are presented as $\mathrm{n}$ or $\mathrm{n}(\%)$, unless otherwise stated. 


\section{TABLE 2 Morphological characteristics of lymph nodes}

\begin{tabular}{|c|c|c|c|}
\hline Morphology & Total & Benign & Malignant \\
\hline Lymph nodes & $740(100)$ & $270(36.4)$ & 463 (62.5) \\
\hline \multicolumn{4}{|l|}{ Shape } \\
\hline Triangular & $50(6.78)$ & 43/50 (89.58) & $5 / 50(10.42)$ \\
\hline Round/oval & 687 (93.22) & $226 / 687$ (33.09) & $457 / 687(66.91)$ \\
\hline \multicolumn{4}{|l|}{ Size } \\
\hline$<10 \mathrm{~mm}$ & $311(42.03)$ & $133 / 311(43.46)$ & $173 / 311(56.54)$ \\
\hline$\geqslant 10 \mathrm{~mm}$ & 429 (57.97) & $137 / 429$ (32.08) & $290 / 429$ (67.92) \\
\hline \multicolumn{4}{|l|}{ Margin } \\
\hline Defined & $668(91.01)$ & $238 / 668$ (35.84) & $426 / 668(64.16)$ \\
\hline Ill-defined & 66 (8.99) & $30 / 66(46.88)$ & $34 / 66$ (53.13) \\
\hline \multicolumn{4}{|l|}{ Central vessel } \\
\hline Present & 102 (13.8) & $71 / 102(71.00)$ & 29/102 (29.00) \\
\hline Absent & $636(85.95)$ & $199 / 636(31.44)$ & $434 / 636(68.56)$ \\
\hline \multicolumn{4}{|l|}{ Echogenicity } \\
\hline Uniform & $664(89.73)$ & $243 / 664$ (36.82) & $417 / 664(63.18)$ \\
\hline Hyper- or hypoechoic & $73(10.27)$ & $26 / 73(36.62)$ & $45 / 73$ (63.38) \\
\hline
\end{tabular}

$11.5 \mathrm{~mm}$ was found to be an independent predictor of malignancy $(\mathrm{p}=0.002)$. Out of 740 nodes, 48 $(6.78 \%)$ nodes were triangular in shape. On analysis $43(88.6 \%)$ nodes with triangular shape were nonmalignant and five (11.4\%) were malignant. The presence of triangular shape was a significant predictor $(p<0.001)$ for a benign aetiology for the lymphadenopathy. A central vessel was present in $103(13.9 \%)$ out of 740 nodes, and $70.5 \%$ of such nodes $(n=71)$ were nonmalignant and $29.5 \%(n=29)$ were malignant. This feature has an independent correlation with a benign aetiology $(\mathrm{p}<0.001)$ (figure 1).

The lymph nodes margins were well-defined on endosonography in 668 (91\%) out of 740 and ill-defined in $68(8.9 \%)$ out of 740 nodes. However, $46.8 \%$ of lymph nodes where the margins were poorly defined were malignant and $53.7 \%$ nodes were nonmalignant. No significant correlation was found between the margin of lymph nodes and final outcome as benign or malignant. Nodes defined as having a uniform echogenicity or heterogeneous echogenicity did not have any clear association with the final outcome.

Of the 153 patients with a final benign outcome ( 270 nodes), 31 patients were diagnosed as sarcoidosis: 25 based on non-necrotising granuloma on EBUS TBNA, four on mediastinoscopy, and two based on clinical picture with other organ involvement and follow-up.

19 patients were diagnosed as having tubercular lymphadenopathy; most of the patients' fine-needle aspiration cytology (FNAC) material contained $M$. tuberculosis. Two patients were acid-fast bacillus-positive on bronchoalveolar lavage and one patient showed changes suggestive of tuberculosis on mediastinoscopy specimens. Two patients' FNAC material contained Actinomyces and one patient's methicillin-resistant Staphylococcus aureus. One patient's lymph nodes regressed on a course of antibiotics.

FIGURE 1 Predictive probability of benign disease by size and presence of central vessels on endobronchial ultrasound.

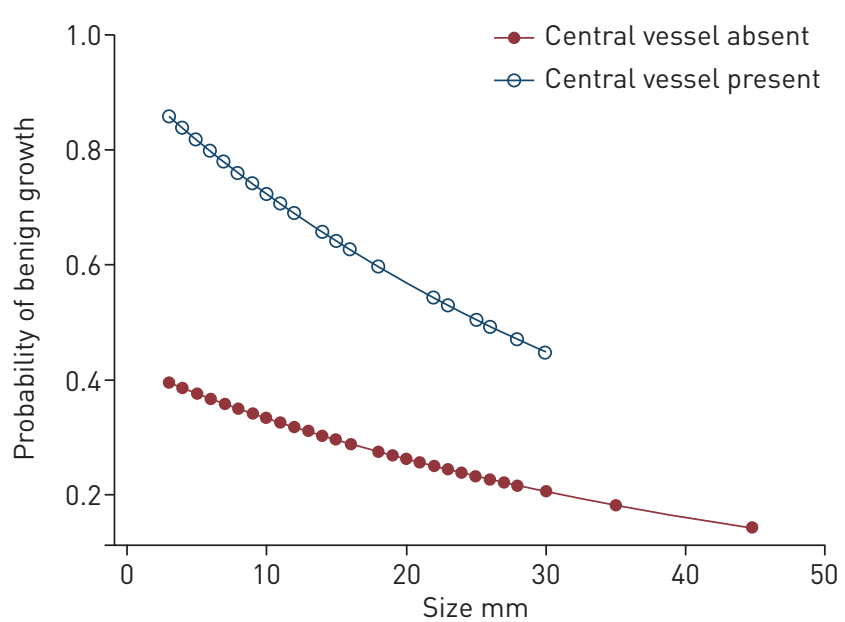




\begin{tabular}{|c|c|c|c|c|c|c|}
\hline & OR $(95 \% \mathrm{CI})$ & AUC & Sensitivity \% & Specificity \% & PPV \% & NPV $\%$ \\
\hline Triangular shape & $17.4(7.0-43.1)$ & 0.575 & 16 & 98.90 & 89.6 & 66.9 \\
\hline \multicolumn{7}{|l|}{ Size } \\
\hline $6 \mathrm{~mm}$ & $1.37(0.93-2.01)$ & 0.523 & 20.4 & 84.2 & 43 & 63.50 \\
\hline $10 \mathrm{~mm}$ & $1.63(1.2-2.2)$ & 0.559 & 49.3 & 62.6 & 43.5 & 67.9 \\
\hline IIl-defined margin & $1.31(0.95-2.63)$ & 0.519 & 11.60 & 92.60 & 46.9 & 64.2 \\
\hline Central vessel present & $5.34(3.37-8.46)$ & 0.6 & 26.3 & 93.7 & 71 & 68.6 \\
\hline
\end{tabular}

AUC: area under the receiver operating characteristic curve; PPV: positive predictive value; NPV: negative predictive value.

Six patients were reported as having reactive lymph nodes. Sufficient lymphocytes for adequate lymph node sampling were observed in all but two samples.

The rest of the patients were assigned benign status based on presence of lymphocytes in FNAC specimens, the absence of malignant cells and adequate follow-up showing nonprogression of disease. 95 (23\%) patients could not have a final diagnosis on EBUS alone and 45 out of these underwent mediastinoscopy. The other 50 patients were followed up for 12 months.

As three out of five parameters studied were independent predictors of a nonmalignant final outcome (table 3), a log-binomial regression analysis was performed considering how any combination of the features size $<10 \mathrm{~mm}$, triangular shape and presence of central vessel predicted benign disease (table 4).

Multivariate logistic regression analysis predicted a risk ratio of benign disease if the size is less than $10 \mathrm{~mm}$ and a central vessel is present (adjusting for shape) as 2.2 , which is statistically significant ( $\mathrm{p}<0.001$ ) (table 5).

A ROC curve was drawn to assess the strength of association of these parameters with nonmalignant outcome and the area under curve was 0.70 , indicating a strong association (figure 2).

\section{Discussion}

Our hospital is a tertiary-care referral centre with an active bronchoscopy unit equipped with EBUS since 2005. We receive EBUS referrals for suspected cancer patients, the staging of cancer in patients with confirmed malignant disease and those patients with mediastinal lymphadenopathy of unknown cause. As the results show, more than one third of our patients had benign or reactive lymphadenopathy. To this end, we sought to classify lymph nodes morphologically and correlate this with the final outcome in order to see which factors predict benign disease. We considered that a combination of morphological findings and FNAC results will give a clinician more confidence in accepting a diagnosis of benign disease if EBUS-TBNA demonstrates adequate sampling but no conclusive cytological features. This may avoid more invasive and potentially unnecessary procedures such as mediastinoscopy, alongside the standard EBUS-TBNA result.

Currently, thoracic CT and PET are used in the evaluation of mediastinal lymphadenopathy. Thoracic CT has a sensitivity of $55 \%$ and specificity of $81 \%$ in identifying malignant disease [15]. PET-CT has a higher sensitivity and specificity than CT but false positives may still occur, particularly in patients with granulomatous disease such as tuberculosis and sarcoidosis [16]. False negatives may also occur, and are more common in lung adenocarcinoma and metastatic disease [17].

\begin{tabular}{|c|c|c|c|c|c|c|}
\hline $\begin{array}{c}\text { Positive } \\
\text { characteristics } \mathrm{n}\end{array}$ & $\begin{array}{c}\text { Size } \\
<10 \mathrm{~mm}\end{array}$ & $\begin{array}{l}\text { Central } \\
\text { vessel }\end{array}$ & $\begin{array}{l}\text { Predictive } \\
\text { probability } \\
(95 \% \mathrm{CI})\end{array}$ & $\begin{array}{c}\text { Sensitivity } \\
\%\end{array}$ & $\begin{array}{c}\text { Specificity } \\
\%\end{array}$ & $\begin{array}{c}\text { NPV } \\
\%\end{array}$ \\
\hline 0 & 0 & 0 & $0.28(0.25-0.32)$ & 35 & 42 & 52 \\
\hline 1 & 1 & 0 & $0.37(0.33-0.41)$ & 39 & 65 & 64 \\
\hline 1 & 0 & 1 & $0.62(0.54-0.71)$ & 16 & 96 & 66 \\
\hline 2 & 1 & 1 & $0.81(0.72-0.91)$ & 10 & 98 & 65 \\
\hline
\end{tabular}




\section{TABLE 5 Relative risk of being benign in final multivariate model}

Characteristics

Size

$<10 \mathrm{~mm}$

$\geqslant 10 \mathrm{~mm}$

Central vessel

Absent

Present
Risk ratio $(95 \% \mathrm{Cl})$

$1.31(1.107-1.549)$

Ref.

Ref.

$222(1.876-2.621)$ p-value

0.002

$<0.001$

Malignant infiltration alters the ultrasonographic features of the cervical lymph nodes, resulting in enlarged nodes that are usually rounded and show peripheral or mixed vascularity [18]. Using these features, ultrasound has been shown to have an accuracy of $89-94 \%$ in differentiating malignant from benign in cervical lymph nodes [18-20]. Similarly, to accurately characterise mediastinal nodes as benign or malignant with endosonographic morphology was the pursuit of this present study.

EBUS morphology of mediastinal lymph nodes in malignant and nonmalignant disease has previously been studied. In a study by FAIGE [21], a morphological visual analogue scale was created evaluating size, shape and echogenicity, and a morphology score was calculated (1: least malignant; 5: most malignant). The results were compared with cytological outcomes and the authors concluded that in malignant adenopathy, lymph nodes are larger, rounder, darker and more homogeneous than those of patients without malignant adenopathy. Our study, where size and shape were found to be important predictors of outcome, is consistent with these results. Triangular shape (figure 3), in particular, is a strong predictor of benign disease. A central vascular structure, though, associated with benign status in cervical lymph nodes has not been previously studied in mediastinal lymph nodes. In our study, there was a very strong correlation with benign status and the presence of a central vessel (OR 5.6). All existing studies emphasise that larger size favours malignant aetiology; however, reactive nodes and those secondary to sarcoidosis may still be large. Different cut-off values of nodal short-axis diameter (5,8 and $10 \mathrm{~mm}$ ) have been used to differentiate malignant from benign diseases; however, a lower cut-off value increases sensitivity but is less specific for malignant aetiology. In a study by GiLL et al. [22], endoscopic ultrasound features in lung cancer-associated mediastinal lymph nodes were size $>8.3 \mathrm{~mm}$, round shape and sharp margins, with a predicted probability of $63 \%$ when all three are present. In our study, the predictive probability of benign disease was $81 \%$ if the size is $<10 \mathrm{~mm}$, a central vessel is present and the node has a triangular shape.

Our study is unique in several aspects, as it has a heterogeneous population, with one third of the patients having nonmalignant pathology. The purpose of the study was not to identify malignant nodes morphologically but was rather to study various nodal appearances and then correlate these with the final outcome. In a study by FüIWARA et al. [11], these parameters were retrospectively analysed to identify metastatic lymph nodes, whilst benign lymph nodes were not studied at all. These authors found a strong correlation of large size, oval shape, defined margins and hypoechoic focus with malignancy.

A limitation of our study is that mediastinoscopy was not performed in all patients with negative FNAC, although all patients were followed up for a significant duration of 12 months. Moreover, benign lymph nodes were not further subdivided as tuberculous, sarcoid associated and benign for finer assessment of

FIGURE 2 Area under curve for endobronchial ultrasound morphological characteristics of node size $<10 \mathrm{~mm}$ with the presence of a central vessel in assessing benign lymph nodes. Area under the curve 0.6716 (SE 0.0249).

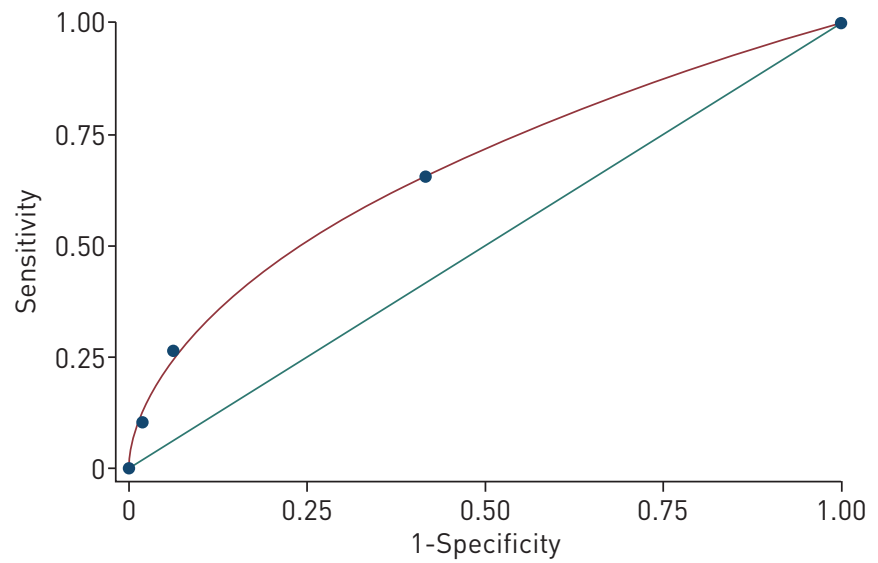


FIGURE 3 Endosonographic image of a triangular node.

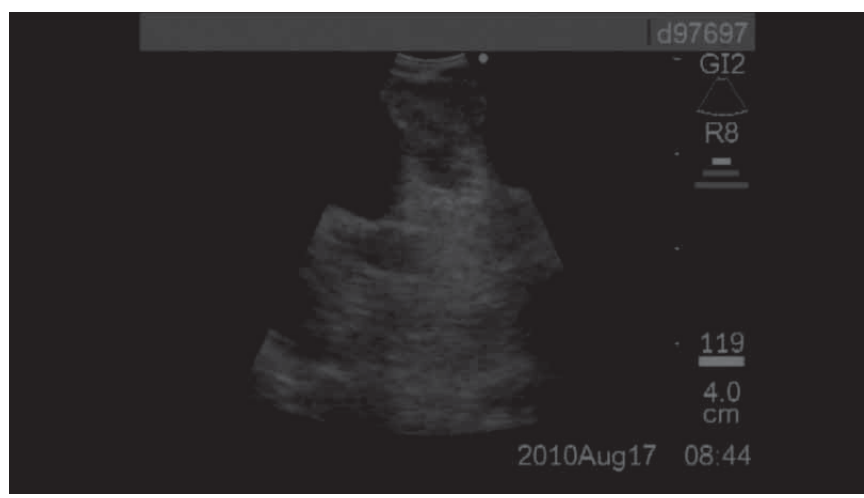

sonographic morphology. A triangular shape was found to have a very strong association with benign cause of mediastinal lymphadenopathy in univariate analysis but needs a more objective definition and a larger sample is needed for applying multivariate correlation. The results from this study should be interpreted with caution as this is a single-centre study. Further research extended to multiple centres is needed to further evaluate the morphological attributes of benign mediastinal lymph nodes.

Recent advances in ultrasound technology, such as contrast-enhanced ultrasound, contrast-enhanced endoscopic ultrasound and real-time elastography show potential to improve the accuracy of ultrasound for the differential diagnosis of benign and malignant lymph nodes [23]. A collective scale can be developed combining thoracic CT, PET-CT, endosonographic morphology and tissue cytology to decide about more invasive options in probable benign causes of mediastinal lymphadenitis.

In conclusion, by carefully evaluating lymph nodes for the endosonographic morphological features of small size $(<10 \mathrm{~mm})$, triangular shape and the presence of a central vessel during EBUS, and using this information in conjunction with benign EBUS-TBNA cytological results, the clinician can more confidently suspect a benign aetiology, potentially avoiding mediastinoscopy or other invasive assessments in selected patients. However, larger studies are needed for further validation of benign lymph nodes features on endosonography.

\section{References}

1 Lema VL, Villar AF, Ruano-Ravina A. Effectiveness and safety of endobronchial ultrasound-transbronchial needle aspiration: a systematic review. Eur Respir J 2009; 33: 1156-1164.

2 Ahuja AT, Ying M. Sonographic evaluation of cervical lymph nodes. Am J Roentgenol 2005; 184: 1691-1699.

3 Kim TH, Kang DK, Kim SY, et al. Sonographic differentiation of benign and malignant papillary lesions of the breast. J Ultrasound Med 2008; 27: 75-82.

4 Lim E, Shah PL. Role of endobronchial ultrasound-guided transbronchial needle aspiration for mediastinal lymph node staging of lung cancer. Thorac Cancer 2010; 1:2-3.

5 Annema JT, van Meerbeeck JP, Rintoul RC, et al. Mediastinoscopy vs endosonography for mediastinal nodal staging of lung cancer: a randomized trial. JAMA 2010; 304: 2245-2252.

6 Tremblay A, Stather DR, Maceachem P, et al. A randomised controlled trial of standard $v s$ endobronchial ultrasonography guided transbronchial needle aspiration in patients with suspected sarcoidosis. Chest 2009; 136: 340-6.16.

7 Von Bartheld MB, Dekkers OM, Szlubowski A, et al. Endosonography vs conventional bronchoscopy for the diagnosis of sarcoidosis: the GRANULOMA randomized clinical trial. JAMA 2013; 309: 2457-2464.

8 Libshitz HI, Mckenna RJ. Mediastinal lymph node size in lung cancer. AJR Am J Roentgenol 1984; 143: 715-718.

9 Henk K, Groen HJM. Current concepts in the mediastinal lymph node staging of nonsmall cell lung cancer. Ann Surg 2003; 238: 180-188.

10 Herth FJ, Eberhardt R, Vilmann P, et al. Real-time endobronchial ultrasound guided transbronchial needle aspiration for sampling mediastinal lymph nodes. Thorax 2006; 61: 795-798.

11 Fujiwara $\mathrm{T}$, Yasufuku $\mathrm{K}$, Nakajima $\mathrm{T}$, et al. The utility of sonographic features during endobronchial ultrasound-guided transbronchial needle aspiration for lymph node staging in patients with lung cancer. Chest 2010; 138: 641-647.

12 Rusch VW, Asamura H, Watanabe H, et al. The IASLC Lung Cancer Staging Project: a proposal for a new International Lymph Node Map in the forthcoming seventh edition of the TNM classification for lung cancer. J Thorac Oncol 2009; 4: 568-577.

13 Barros AJ, Hirakata VN. Alternatives for logistic regression in cross-sectional studies: an empirical comparison of models that directly estimate the prevalence ratio. BMC Med Res Methodol 2003; 3: 21.

14 McNutt LA, Wu C, Xue X, et al. Estimating the relative risk in cohort studies and clinical trials of common outcomes. Am J Epidemiol 2003; 157: 940-943.

15 Kumar A, Dutta R, Kannan U, et al. Evaluation of mediastinal lymph nodes using ${ }^{18}$ F-FDG PET-CT scan and its histopathologic correlation. Ann Thorac Med 2011; 6: 11-16.

16 Sanli M, Isik AF, Zincirkeser S, et al. Reliability of positron emission tomography in identification of mediastinal lymph node status in patients with non-small cell lung cancer. J Thorac Cardiovasc Surg 2009; 138: 1200-1205. 
17 Hwangbo B, Kim SK, Lee HS, et al. Application of endobronchial ultrasound-guided transbronchial needle aspiration following integrated $\mathrm{PET} / \mathrm{CT}$ in mediastinal staging of potentially operable non-small cell lung cancer. Chest 2009; 135: 1280-1287.

18 Griffith JF, Chan AC, Ahuja AT, et al. Neck ultrasound in staging squamous oesophageal carcinoma: a high yield technique. Clin Radiol 2000; 55: 696-701.

19 Kouvaraki MA, Shapiro SE, Fornage BD, et al. Role of preoperative ultrasonography in the surgical management of patients with thyroid cancer. Surgery 2003; 134: 946-954.

20 Ying M, Ahuja A, Metreweli C. Diagnostic accuracy of sonographic criteria for evaluation of cervical lymphadenopathy. J Ultrasound Med 1998; 17: 437.

21 Faige DO. EUS in patients with benign and malignant lymphadenopathy. Gastrointestendosc 2001; 53: 593-598.

22 Gill KRS, Ghabril MS, Jamil LH, et al. Endoscopic features predictive of malignancy on FNA in lung cancer associated mediastinal lymph nodes. Gastrointest Endosc 2010; 72: 265-271.

23 Cui X-W, Jenssen C, Saftoiu A, et al. New ultrasound techniques for lymph node evaluation. World J Gastroenterol 2013; 19: 4850-4860. 